\title{
Comprehensive Review of Molecular Mechanisms during Cholestatic Liver Injury and Cholangiocarcinoma
}

Shohaib Virani ${ }^{3}$, Austin Akers ${ }^{2}$, Kristen Stephenson ${ }^{2}$, Steven Smith ${ }^{2}$, Lindsey Kennedy ${ }^{3}$, Gianfranco Alpini ${ }^{1,3}$ and Heather Francis ${ }^{1,3}$

${ }^{1}$ Research, Central Texas Veterans Health Care System, Texas, USA

${ }^{2}$ Department of Internal Medicine, Baylor Scott \& White Health, Texas, USA

${ }^{3}$ Department of Medical Physiology, College of Medicine Texas A\&M Health Science Center, Temple, Texas, USA

${ }^{*}$ Corresponding author: Heather Francis, Associate Professor, Department of Medical Physiology, College of Medicine,Texas A\&M University, Central Texas Veteran's Health Care System, Texas, USA, Tel: +254-743-1048; E-mail: hfrancis@medicine.tamhsc.edu / Heather.Francis@VA.GOV

Received date: August 23, 2018; Accepted date: September 17, 2018; Published date: September 20, 2018

Copyright: (c) 2018 Virani S, et al. This is an open-access article distributed under the terms of the Creative Commons Attribution License, which permits unrestricted use, distribution, and reproduction in any medium, provided the original author and source are credited.

\begin{abstract}
Cholestatic liver injury is characterized by damage induced on the biliary tree and cholangiocytes, the cells lining the biliary tree, thus they are termed "cholangiopathies". Cholangiopathies include diseases such as Primary Biliary Cholangitis, Primary Sclerosing Cholangitis, Biliary Atresia and Cholangiocarcinoma. These pathologies lack viable therapies and most patients are diagnosed during late stage disease progression (with the exception of Biliary Atresia, which is found shortly after birth). The lack of therapies for these diseases has put a significant burden on the need for liver transplantation as this is the only indicative "cure" for cholangiopathies. The molecular mechanisms for cholangiopathies have been extensively studied; however, and unfortunately, the lack of effective biomarkers and therapeutics remains. In this review article we highlight the latest studies to investigate the molecular mechanisms regulating cholangiopathies and the potential therapeutics that might be discovered.
\end{abstract}

\section{Keypoints}

Cholangiopathies are diseases that target cholangiocytes with limited therapeutic options/treatments. To date, liver transplantation is the only effective "cure" for cholangiopathies.

The molecular mechanisms regulating cholangiopathies has been widely investigated, yet there remains a lack of biomarkers and therapies.

Most cholangiopathies present at a later, progressed state of injury (except for Biliary Atresia, which is found shortly after birth) making patient outcomes dismal.

Translational studies are lacking and significantly warranted to uncover potential therapeutic avenues.

Keywords Cholangiopathies; Therapy; Molecular mechanisms

Abbreviations PBC: Primary biliary cholangitis; AMA: Antimitochondrial antibody; UDCA: Ursodeoxycholic acid; ALP: Alkaline phosphatase; AST: Aspartate transaminase; NK: Natural killer; E. coli: Escherichia coli; UC-MSC: Umbilical cord-derived mesenchymal stem cell; TE: Transient elastography; UC: Ulcerative colitis; PSC: Primary sclerosing cholangitis; IDO: Indoleamine 2,3 dioxygenase; TNF: Tumor necrosis factor; LSM: Liver stiffness measurement; IBD: Inflammatory bowel disease; MRCP: Magnetic resonance cholangiopancreatography; ERCP: Endoscopic retrograde cholangiopancreatography; PTC: Percutaneous trans-hepatic cholangiography; SASP: Senescence-associated secretory phenotype; TLR: Toll-like receptor; INF: Interferon; VAP-1: Vascular adhesion protein 1; MAdCAM-1: Mucosal addressin cell adhesion molecule 1; Mdr2: Multidrug resistance gene 2; Mdr2-/-: Mdr2 knock out; HR: Histamine receptors; BA: Biliary atresia; HDC-1: Histidine decarboxylase; MAOB: Monoamine oxidase B; CCA: Cholangiocarcinoma; IL-6: Interleukin 6; Mcl-1: Myeloid cell leukemia-1; STAT3:
Signal transducers and activators of transcription 3; FGFR: Fibroblast growth factor; PDGFR: Platelet-derived growth factor receptor; LNAME: Nitro-L-arginine methyl ester hydrochloride; HDC: Histidine decarboxylase.

\section{Primary Biliary Cholangitis}

\section{Introduction}

Initially described in 1851, primary biliary cirrhosis, now known as primary biliary cholangitis (PBC), is a chronic autoimmune disease seen primarily in middle-aged women [1]. $\mathrm{PBC}$ results in progressive destruction of intrahepatic bile ducts causing subsequent chronic cholestasis, portal inflammation, and fibrosis, which can ultimately lead to cirrhosis and hepatic failure [2]. The clinical presentation of PBC is comprised primarily of pruritis, fatigue and other generalized symptoms. Ultimately, the diagnosis is based on two out of the three following criteria: the presence of biochemical cholestasis (an elevated ALP), presence of ant-mitochondrial antibodies (AMA), and classic histological findings of $\mathrm{PBC}$ on a liver biopsy (destructive cholangitis affecting the interlobular bile ducts) [3,4]. In a T2-weighted MRI, the classical presentation of a liver with PBC would show fibrosis of the parenchyma of the liver with a periportal halo sign. Other signs could include periportal hypersensitivity, regional lymphadenopathy and splenomegaly [5]. The prevalence of $\mathrm{PBC}$ ranges between 6.7 and 402 cases per million persons, while the annual incidence ranges between 0.7 and 49 cases per million persons [6]. Patients with PBC typically range in presentation from asymptomatic to fulminant liver failure necessitating liver transplant [7]. Most commonly, patients present with pruritus and fatigue as well as jaundice, xanthomas, osteoporosis, and dyslipidemia [8]. PBC affects approximately 9 females to 1 male and is multifactorial in etiology including genetic predisposition and environmental insults [9]. Elevated anti-mitochondrial antibody 
(AMA) is the hallmark of the disease and is present in greater than $90 \%$ of individuals with the condition [1]. AMA has replaced the need for liver biopsy in order to make a diagnosis of PBC [7]. Despite major medical advances, there remains only one FDA approved medication for the treatment of PBC, ursodeoxycholic acid (UDCA) [1]. UDCA is typically prescribed at a dose of $13-15 \mathrm{mg} / \mathrm{kg}$ body weight. Higher doses in patients with inadequate response have shown to be ineffective and, in a few cases, higher doses may be more damaging $[1,7]$. Studies have demonstrated improved transplant-free survival in patients on UDCA monitored at one year with reduction of alkaline phosphatase (ALP) and aspartate transaminase (AST) to $<1.5$ times the upper limit of normal, and a normal total bilirubin after one year of treatment [1]. Figure 1 describes the presentation, investigation and histological appearance of PBC.

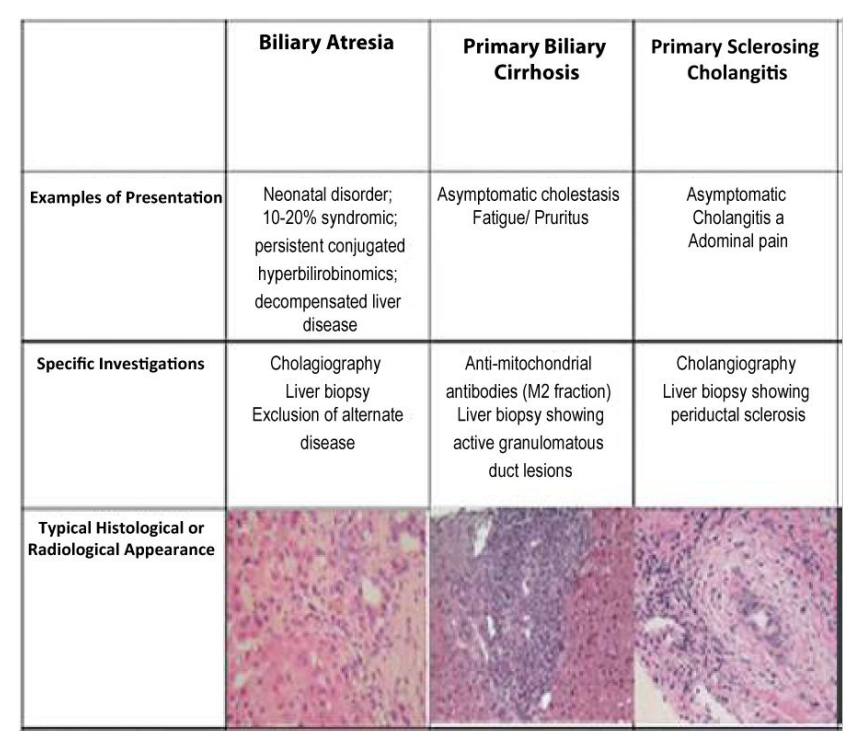

Figure 1: Presentation, investigation and typical histological appearance of BA, PBC and PSC. Modified and reprinted with permission from: Hirschfield G.M. (2014) The Diagnosis and Classification of Immune-Mediated Biliary Diseases. In: Gershwin M., Vierling J., Manns M. (eds) Liver Immunology. Springer, Cham.

\section{Molecular mechanism of injury}

PBC is largely suspected to be secondary to an autoimmune process involving the liver, which leads to destruction of small and mediumsized bile ducts. Histological findings include damaged cholangiocytes and infiltration of the portal area with $\mathrm{B}$ cells, $\mathrm{T}$ cells, macrophages, eosinophils, and natural killer (NK) cells increasing the risk for cirrhosis and hepatic failure [6]. Sjogren syndrome, autoimmune thyroid disorders, and Raynaud syndrome as well as urinary tract infections have been linked with PBC. An epidemiological study showed that $48 \%$ of $\mathrm{PBC}$ patients experienced prior recurrent urinary tract infections versus $31 \%$ of controls [10]. Most commonly, urinary tract infections in women are caused by Escherichia coli (E. coli) prompting its study in relation to PBC. E. coli infections are suspected to cause molecular mimicry of $E$. coli proteins with human dehydrogenase complex leading to the induction of B- and T-cell responses and subsequent autoimmune responses typically found in PBC [8].
Animal models have demonstrated that the etiology of PBC is multifactorial requiring both environmental insults and genetic predisposition in order to break tolerance and lead to eventual liver pathology [9]. These models have shown that NKT cells play an important role in the initiation of disease in the serologically positive patients, while $\mathrm{CD}^{+}$memory $\mathrm{T}$ cells are directly involved in the destruction of cholangiocytes [9].

Reactive oxygen species are produced via many microscopic processes that take place within the cells $[11,12]$. These reactive molecules induce DNA damage and initiate protein degradation [11]. Nitric oxide-derived oxidative kills pathogens, mediate the immune response, and lead to cellular damage [13]. Nitric oxide-derived oxidative species lead to prolonged interruption of the circulation of bile acids, ultimately leading to cholestasis and the induction of PBC [14].

\section{New therapeutic targets}

UDCA has not been shown to improve all-cause mortality, pruritis, fatigue, or outcomes from liver transplantation, but it was found to have a beneficial effect on the histological examination [15]. For patients who do not respond to UDCA, there are currently no alternative treatments that delay the progression of PBC [1]. Liver transplant continues to be the definitive treatment for advanced disease, with an approximate 70\%, 10-year survival following transplantation [8]. Post-transplant recurrence occurs in approximately $30 \%$ at 10 years and $40 \%$ at 15 years, which is often a challenging diagnosis given that AMA remains persistently positive in most patients [8].

Obetocholic acid is a farsenoid-X-receptor agonist that is currently being studied when given in addition to UDCA. The semi-synthetic analogue of chenodeoxycholic acid is present in the liver, kidneys, adrenal glands, and intestine. It exerts its action on 7 alpha hydroxylase leading to decreased bile acid uptake proteins and increased expression of bilirubin exporter pumps, thereby reducing bile acid synthesis and reducing their toxic effects $[1,7,8]$.

Pruritis is the predominant complaint of patients suffering from cholestatic liver disease [16]. Cholestatic itch has long been countered by the cholestyramine treatment, which works by reducing bile acid reabsorption [17]. For patients who do not respond to cholestyramine or who cannot tolerate its side effects, plasmapharesis remains an option [7]. Previous studies have shown that plasmapharesis is a method for treating refractory pruritus but further studies are needed to establish when this option should be utilized [7].

Umbilical cord-derived mesenchymal stem cell (UC-MSC) transfusion has been studied in order to delay or prevent $\mathrm{PBC}$ progression in patients who did not respond to UDCA. It is thought that UC-MSC may suppress the antigen-induced autoimmune condition, as well as stimulate repair of the injured bile ducts. In addition, US-MSC was shown to improve quality of life in PBC patients as it alleviated pruritus and fatigue [18].

\section{Future outlook on the disease}

Current studies show that indoleamine 2,3 dioxygenase (IDO) could play a potential role in PBC. IDO is an intracellular enzyme, which functions as an immunosuppressant. It is thought that impaired IDO expression is involved in the progress of autoimmunity in PBC. The effects that IDO exerts on tryptophan and its catabolism could 
contribute to potential treatment opportunities and biomarkers for disease progression [19].

Liver stiffness measurement (LSM) is currently being studied, as there are no reliable markers of liver fibrosis in PBC. Transient elastography (TE) was studied in a large cohort to monitor UDCAtreated patients and noninvasively assess liver stiffness. As there are no current serum surrogate markers of liver fibrosis routinely monitored in those with PBC, LSM could play an important role for clinicians to evaluate treatment. Monitoring of TE provided important prognostic information for PBC patients, in particular, those with cirrhosis and may be of benefit to predict outcomes. It was also associated with elevated levels of hyaluronic acid, which may be useful to monitor in PBC patients. Confirmation of these results is needed with larger studies, though improved monitoring in regards to response to treatment may be on the horizon [20].

Levels of albumin, ALP, and bilirubin have been studied as surrogate endpoints in therapy trials. Survival of patients with advanced disease and biochemical response to UDCA has significantly improved for those without response. There is discussion whether albumin and bilirubin are superior to the information obtained from ALP. In addition, questions remain regarding the appropriate time to monitor for improvement of these markers at either 6 months or 1 year $[21,22]$.

There are several promising alternatives such as obetocholic acid, which are still undergoing trials. Appropriate monitoring concerning response to treatment also has questions to be answered as to whether liver function tests such as ALP, albumin, and bilirubin are as accurate as TE. Ideally, future studies will continue to solve the mystery of PBC's mechanism of action and identify new information pertaining to IDO that may play a role in treatment and possibly decrease the number transplants. Post-transplant recurrence requires additional studies, as there is no current guideline directed therapy for these patients.

\section{Primary Sclerosing Cholangitis}

\section{Introduction}

Primary sclerosing cholangitis (PSC) involves chronic inflammation, fibrosis, and eventual destruction of the intra- and extra-hepatic biliary system $[23,24]$. PSC is typically diagnosed in the third through fifth decades of life and has an approximately 3 to 1 male to female predilection. Diagnosis of PSC after the age of 50 is considered late onset and one study showed that late onset PSC was associated with more frequent dilatation therapy, recurrent cholangitis, and decreased transplant-free survival when compared to those diagnosed with PSC before age 50 [25]. PSC is a relatively rare disease and while the incidence is not well established, a systematic review of 8 studies from North America and Europe in 2011 showed an overall incidence of 0.77 per 100,000 person years [26]. The prevalence data has varied and even the data on the higher end of the spectrum reveals a prevalence of only 6 per 100,000 individuals [27]. Figure 1 describes the presentation, investigation and histological appearance of PSC.

There is a strong association between PSC and inflammatory bowel disease (IBD), particularly ulcerative colitis (UC). Studies have consistently shown that greater than $70 \%$ of PSC patients have concurrent IBD, the vast majority of which will be UC, and 5-7.5\% of UC patients will eventually be diagnosed with PSC. Thus, patients diagnosed with PSC are recommended to routinely undergo screening for IBD. Individuals with PSC and IBD have been shown to have a significantly higher probability of developing colorectal cancer or cholangiocarcinoma $[28,29]$. One study showed that patients with both PSC and IBD carried a two-fold increased risk of developing advanced colorectal neoplasia compared to patients with only IBD [29]. Furthermore, PSC is the most significant known risk factor for cholangiocarcinoma and these patients carry a lifetime risk of 10 to 15 percent [30-32]. PSC is also a risk factor for other hepatobiliary cancers including hepatocellular carcinoma and gallbladder carcinoma. Ali et al. demonstrated that approximately $9.5 \%$ of patients with PSC over a 20 -year time period developed hepatobiliary cancer [33]. Of those, 78\% developed cholangiocarcinoma, 21\% developed hepatocellular carcinoma, and 6\% developed gallbladder carcinoma [33]. Given the risk of developing hepatobiliary cancer, the AASLD recommends surveillance for hepatobiliary cancer with an annual ultrasound. One study showed that patients with PSC who underwent surveillance for hepatobiliary cancer had significantly higher 5-year survival (68\% vs. $20 \%$, respectively) [33]. Survival times vary considerably based on the stage of disease at the time of diagnosis, with one study showing a median survival time from diagnosis to either death or liver transplantation of 9.6 years [30].

About half of the patients suffering from PSC are asymptomatic at the time of diagnosis, despite having advanced stages of the disease. The most common symptoms that symptomatic patients present with include fatigue and pruritis [34,35]. Elevated liver enzymes in an obstructive pattern are the most common laboratory finding, with a predominantly elevated ALP level [36]. A large portion of PSC patients are positive for $\mathrm{p}$-ANCA, anti-SM and ANA antibodies, however these antibodies have low specificity for PSC and frequencies vary between studies [37]. PSC can be diagnosed on cholangiography with findings of bile duct strictures with segmental dilation, giving the appearance of "beading" [38]. Cholangiography is obtained via magnetic resonance cholangiopancreatography (MRCP), endoscopic retrograde cholangiopancreatography (ERCP), or percutaneous trans-hepatic cholangiography (PTC) [39]. As the least invasive method, MRCP carries minimal risk $[36,39]$. Liver biopsy is usually unnecessary for diagnosis and its utility in the evaluation of liver disease is declining $[36,39]$.

There is currently no FDA approved pharmaceutical treatment for PSC. UDCA has been the most extensively studied treatment, and although it has been shown to reduce ALP levels, evidence of clinical improvement and prolonged survival is lacking. Lindor et al. showed that UDCA at doses of 13 to $15 \mathrm{mg}$ per $\mathrm{kg}$ of body weight per day resulted in improvement in ALP, AST, bilirubin, and albumin levels but no significant difference in time to treatment failure or time to liver transplantation [40]. This study further showed that serious adverse effects including death, the development of varices, and liver transplant eligibility occurred more often in the group treated with UDCA [41]. Additionally, meta-analysis by Triantos et al. showed that when comparing standard or high dose UDCA to placebo or no intervention there was no significant difference outcome in regards to symptoms or progression of disease [42]. Given the lack of clinical efficacy and potential for adverse effects, the use of UDCA remains controversial. ACG guidelines from 2015 do not recommend for or against using UDCA in the treatment of PSC.

Medical therapy of PSC that involves altering the gut microbiota is of increased interest. One case report showed that four weeks of daily fecal microbiota transplant enemas in a patient with UC and PSC resulted in improvement of IBD symptoms and normalization of liver enzymes [43]. Further research is needed to determine whether normalization of liver enzymes correlates with clinical improvement. 
Vancomycin has been studied in PSC treatment and a case study showed a 12-year-old female with PSC status post liver transplant was treated with oral vancomycin $500 \mathrm{mg}$ three times daily [44]. With this treatment, liver enzymes normalized and repeat liver biopsy with three years of continued treatment showed no evidence of bile duct inflammation [44].

The only definitive treatment of PSC is liver transplantation; however, this is only indicated in a subset of patients. Indications include recurrent bacterial cholangitis, decompensated cirrhosis, hepatocellular carcinoma, hilar cholangiocarcinoma and sequelae of portal hypertension. Among patients who receive liver transplantion, clinical recurrence after transplant remains a possibility. A systematic review by Gautam et al. showed a recurrence rate after liver transplant of 11 percent [45]. Another study showed that over time, risk of recurrence increases from $2 \%$ at 1 year to as high as $20 \%$ at 10 years, and those who were diagnosed with cholangiocarcinoma prior to initial liver transplant carried a significantly higher risk of recurrence [46].

\section{Molecular mechanisms of injury}

The pathological mechanisms of PSC are incompletely understood and likely multifactorial. Intestinal microbial dysbiosis is one proposed mechanism, given the poorer outcomes (shorter transplant-free and overall survival) and higher rates of cholangiocarcinoma amongst those with concurrent IBD and PSC [47]. The translocation of intestinal bacteria across an inflamed, permeable gut combined with decreased proliferation of other beneficial organisms may result in an overall increased immune response to intestinal endotoxins $[48,49]$.

The concept of cellular senescence and the senescence-associated secretory phenotype (SASP) is an emerging mechanism of cholangiocyte damage and destruction in PSC [50]. This phenotype is associated with increased pro-inflammatory chemokines, cytokines, and extracellular matrix remodeling proteases. Overall, PSC cholangiocytes differ significantly from normal human cholangiocytes. They exhibit features such as "diminished monolayer formation, barrier function, and associated tight junction protein expression, as well as impaired growth and upregulation of inflammatory signaling molecules," as demonstrated in a 2014 study which characterized cultured PSC cholangiocytes [51]. SASP is linked with dysbiosis, as UDCA is a commensal gut bacteria metabolite which abrogates cholangiocyte senescence in vitro and, when absent in animal models, results in cellular senescence and exacerbated biochemical and histological features of PSC [51].

Additionally, disruptions in biliary epithelial cell tight junctions may expose cholangiocytes to a variety of substances, which can activate an inflammatory response. These disruptions are mediated by toll-like receptor (TLR) mechanisms and inflammation is induced by a variety of molecular mediators, which ultimately leads to myofibroblast activation and fibrosis. As discussed in a 2013 article, in liver explants from patients with PSC, biliary epithelial cells express higher levels of TLR, nucleotide-binding oligomerization domain, the MyD88/IRAK complex, tumor necrosis factor (TNF) $\alpha$, interferon (INF) $\gamma$, and IL8, than cells from individuals without PSC [52].

\section{New therapeutic targets}

$\mathrm{N}$-Ras is a known inducer of cellular senescence and is increased in PSC cholangiocytes 50. Additionally, experimentally induced senescent human cholangiocytes have been shown to cause senescence in bystander cholangiocytes. Targeted therapy towards this protein and other inducers of cellular senescence could have significant utility in the treatment of PSC.

One of the proposed mechanisms by which PSC and IBD are linked is the upregulation of vascular adhesion protein 1 (VAP-1) on mesenteric vessels. VAP-1 induces the generation of mucosal addressin cell adhesion molecule 1 (MAdCAM-1) and the chemokine CCL25 in the gut and on liver sinusoidal endothelium. The expression of these adhesion molecules and chemokines is increased in the PSC liver as well. Ultimately this results in the increased lymphocyte recruitment to the liver and biliary tree, resulting in inflammation and cellular destruction [53]. Agents targeting these and other adhesion molecule/ chemokine interactions are currently undergoing trials for the treatment of IBD and may show future utility in PSC treatment.

Existing evidence in animal models suggests that 24-norUDCA, a C23 homologue of UDCA, is superior to UDCA in improving AP and ALT levels, markers of inflammation, and liver histology [53]. As it is primarily secreted in the unchanged glucuronidated form, 24norUDCA increases hydrophilicity of biliary bile acids stimulating bile flow. This increased elimination decreases cholangiocyte inflammatory response. Phase II clinical trials in humans have yielded favorable results, with evidence that 24-norUDCA dramatically decreases ALP levels in a dose-dependent manner [54]. Additionally, it has been shown to have a favourable safety profile with minimal side effects, and these results warrant further evaluation with phase III trials.

Mast cells have been indicated to play a role in many processes of hepatic injury [55,56]. Following hepatic injury, mast cells release migrates to the liver and is activated, thereby releasing increased histamine which stimulates biliary proliferation and hepatic fibrosis [57]. Mast cell migration is proportional to the progression of liver disease, and mast cells are found in close proximity to bile ducts following injury [55,57]. The multidrug resistance gene $2(\mathrm{Mdr} 2)$ is an adenosine triphosphate-binding cassette transporter, which encodes a P-type glycoprotein responsible for the excretion of biliary phospholipids [58]. Damage in the livers of mice with the Mdr2 knocked out (Mdr2-/-) have been shown to mimic PSC-associated damage [59]. In Mdr2-/-, inhibition of mast cell-secreted histamine via cromolyn sodium treatment decreases biliary proliferation and hepatic fibrosis, highlighting the therapeutic potential for this compoun [58]. In a recent study by Kennedy et al. (Figure 2), the authors treated Mdr2-/- mice with over the counter antihistamines (blocking H1 histamine receptors (HR) or H2HR) for 4 weeks and found an amelioration of PSC-induced biliary damage, inflammation and hepatic fibrosis [60]. Mice were given treatments either separately or in combination and similar results were found [45]. These studies point to potential translational therapies for patients suffering from PSC since these drugs are readily available.

Immunosuppressive agents have traditionally not been shown to offer any mortality benefit in PSC. A meta-analysis and systemic review looked at several immunosuppressive agents in the treatment of PSC and found no survival benefit or decreased necessity of liver transplantation [61]. Conversely, four children with PSC were treated with mizoribine and azathioprine, immunosuppressants, and significant benefits were noted [62]. 


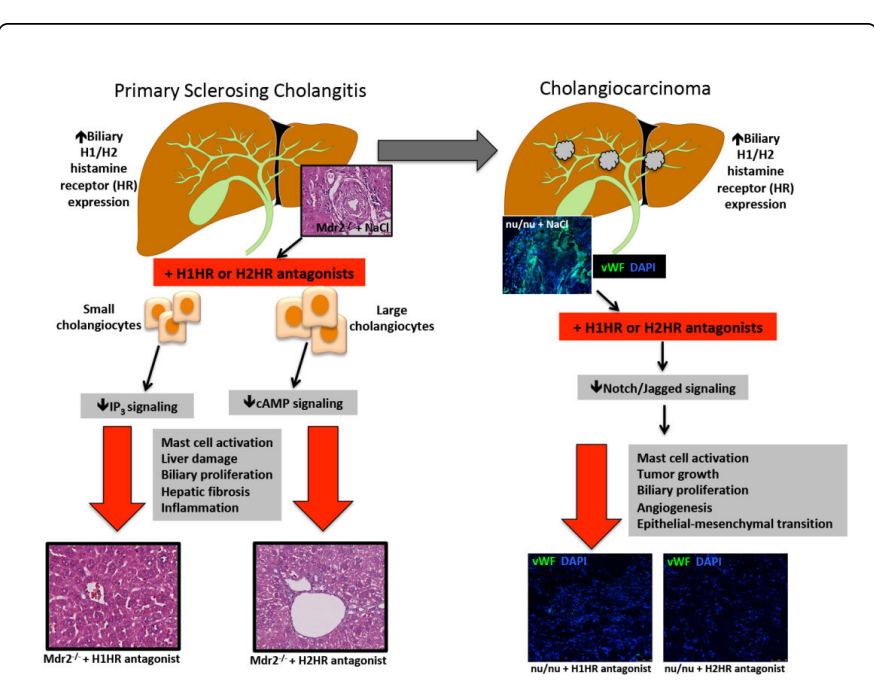

Figure 2: PSC can progress to cholangiocarcinoma (CCA). This study reveals that the $\mathrm{H} 1$ and $\mathrm{H} 2 \mathrm{HRs}$ are upregulated in both mouse models and human PSC and CCA. (LEFT) Blocking H1HR decreases small cholangiocyte proliferation via IP3 signaling, whereas H2HR decreases large cholangiocyte proliferation. Inhibition of either H1HR or H2HR or a combination of these drugs reduces overall liver damage, mast cell activation, biliary proliferation, hepatic fibrosis and inflammation. (RIGHT) Treatment with $\mathrm{H} 1 \mathrm{HR}$ or H2HR inhibitors in nu/nu mice with xenograft CCA tumors results in decreased mast cell activation, tumor growth, biliary proliferation, angiogenesis and epithelial to mesenchymal transition via decreased Notch/Jagged signaling.

Two of the four children were treatment naive patients and two had established liver cirrhosis. The treatment naive patients were found to have normalization of liver enzymes following treatment and patients with established liver cirrhosis had improvement in liver histology as well as MRCP findings with the same treatment [62]. This combination therapy would need to be further studied with a much larger cohort to prove clinical significance. Additionally, a case report by Tischendorf et al. showed radiographic and laboratory improvement in a patient with PSC after a 13 month course of the immunosuppressant vedolizumab [63]. The patient had clinical improvement with reduction in pruritus and abdominal pain secondary to PSC. Success with immunosuppressant medications should call into question the diagnosis, as autoimmune cholangiopathy presents with overlapping features of PBC/PSC and autoimmune hepatitis $[64,65]$.

\section{Biliary Atresia}

\section{Introduction}

Biliary atresia (BA) is characterized by fibrosis of the biliary tract in newborn infants that leads to obstruction of the extrahepatic bile ducts and subsequent liver cirrhosis indicative of liver transplant [66,67]. BA is a disease process that comprises anywhere from $34 \%-42 \%$ of neonatal jaundice [68]. The prevalence of BA was estimated to be 0.5 to 0.8 per 10,000 live births in Western countries [69]. The incidence of BA in the US was found to be 4.47 per 100,000 and was found to be higher in females (risk ratio of 1.43), and in Asian/Pacific Islander (risk ratio of 1.89) and African Americans (risk ratio of 1.30) as compared to Caucasians [70]. An infant suffering from BA would likely present with jaundice, polyspenia, vascular anomalies, situs inversus and cardiac abnormalities [71]. An ultrasound of the liver in patients suffering from BA would show echogenic fibrous tissue anterior to the portal vein, also known as the triangular cord sign [72]. The stool color card is the method that has gained the most attention due to its ease of implementation and non-invasive nature. The stool color card is the norm of diagnosis and contains photographs of normal and abnormal appearing stools. Use of the card, given to a postpartum mother, was found to reduce the amount of time that was taken before an infant was given treatment from approximately 70 days to 59.7 days. However, the card had a positive predictive value of only $12.7 \%$, meaning that many infants that produced a positive test did not actually have BA [73]. The current treatment of BA is to re-establish bile flow via the use of the Kasai procedure, a portoenterostomy. This procedure, named after Dr. Morio Kasai, is preceded by the removal of the damaged bile ducts. Figure 1 describes the presentation, investigation and histological appearance of BA.

\section{Molecular mechanisms of injury}

Studies have identified many factors in the pathogenesis of BA. In a 2014 study, a neonatal mouse model of rotavirus (RRV)-induced BA identified perforin and granzymes, released from NK cells and CD8+ T cells, may work synergistically to lead to cholangiocyte apoptosis [74]. When each of these molecular mechanisms was inhibited independently, cell lysis was miniscule. However, it was demonstrated that experimental BA could be successfully prevented by inhibition of both granules [74].

A 2013 human study used immunohistochemistry on resected bile ducts and liver biopsy specimens from 21 patients with BA ( 9 male/12 female; average age of 1.7 months) and compared them to specimens from one neonatal hepatitis patient and 6 patients with nonhepatobiliary diseases [75]. This study found that BA patients were found to have increased levels of interleukin-32 induced by biliary innate immunity via toll-like receptor 3 and other pro-inflammatory cytokines [75].

Studies that focused on the molecular mechanism of BA in mice have also found some promising information. In a 2017 study, TNFa and the expression of its receptors (TNFR1 or TNFR2) had been linked to apoptosis in bile duct epithelial cells [76]. The authors found that overexpression of TNFa by NK cells induced lysis of $55 \% \pm 2 \%$ of cholangiocytes. Neonatal mice using the rotavirus (RRV)-induced BA model were given an antibody against $\mathrm{TNF}$, to block its downstream effects, or an antibody against TNFR1 or TNFR2 to block the binding of TNFa. The authors found that blocking TNFa or TNFR2 resulted in complete prevention of cholangiocyte lysis [76].

Histamine is a major inflammatory mediator that plays a role in cell proliferation, inflammation, extracellular matrix deposition and cellmatrix interactions [77]. Histamine has been linked to the migration of fibroblasts and the generation of hepatic fibrosis [77,78]. Finally, histamine has been found to play a role in the pathogenesis of BA. A recent study found that $B A$ patients have increased hepatic histamine levels, which positively correlated with hepatic fibrosis. Additionally, hepatic 1-histidine decarboxylase (HDC, synthesizes histamine) expression was increased, while monoamine oxidase B (MAOB, breaksdown histamine) was reduced in BA patients [79]. These findings corroborate what has previously been known about the profibrogenic role of hepatic histamine signalling [79]. 


\section{New therapeutic targets}

Recently, surgeons have attempted to complete the previously discussed Kasai procedure in a laparoscopic manner [80]. A 2015 meta-analysis study compared an open portoenterostomy with the laparoscopic procedure and found that there was no statistically significant difference in operation time, hospital stay, intraoperative blood loss, cholangitis, or variceal bleeding between the two methods. Furthermore, 2-year survival (barring liver transplant) was higher in patients who elected for the open procedure [81]. On the contrary, Pediatric Surgery International published an article in which they reevaluated the minimally invasive surgical option. There were 22 cases of BA treated using the laparoscopic portoenterostomy between 2009 and 2016 which indicated that the use of this minimally invasive method resulted in the use of fewer anesthetics, less respiratory support and a minimal incidence of postoperative morbidities [82]. Since the advances of this procedure are new, it is clear that there will continue to be improvements in the logistics and quality of the operation.

In terms of BA prevention, a study involving the investigation of zebrafish who were exposed to factors that could potentially induce BA, found some groundbreaking news [83]. These fish were introduced to a previously unidentified isoflavonoid, biliatresone, 5 days after fertilization and were then studied for changes in bile ducts and gallbladders. This compound selectively destroyed extrahepatic bile ducts and was implicated in the destruction of cilia and disruption of cell polarity in neonatal mice. Furthermore, isofavonoids are currently found in many commonly eaten foods, such as chard, table beet, and sugar beet [83]. This is evidence that exposure to environmental factors during gestation does affect BA development, which could lead to promising actions that can be taken towards prevention of BA during the developmental stages of pregnancy.

Looking forward, there are many genes and proteins that may play a role in the pathophysiology of BA. GATA6 is a transcription factor that was found to be positively associated with cases of lung adenocarcinoma and is a possible target in the inhibition of progression of human laryngeal squamous cell carcinoma $[84,85]$. In terms of BA, GATA6 was found to have an elevated level of expression in cholangiocytes and hepatocytes in neonatal livers suffering from BA [86]. Additionally, completion of successful portoenterostomy downregulated the expression of GATA6 in these cells [86].

\section{Cholangiocarcinoma}

\section{Introduction}

Cholangiocarcinoma (CCA) is a cancer that arises from the epithelial cells lining the bile ducts [87]. CCA is usually divided into two subtypes based on anatomical location: intrahepatic and extrahepatic. Within the extrahepatic category, there is perihilar and distal CCA [88]. A study including 564 patients between 1973 and 2004 found that the percentage of perihilar disease was approximated to be around $50 \%$, while the percentage of distal CCA was found to be near $40 \%$, leaving less than $10 \%$ of CCA cases to be classified as intrahepatic cases [89]. In terms of the incidence of these two subtypes of CCA in the United States, intrahepatic CCA has seen an increase in cases from 0.44-1.18 cases per 100,000 person-years between 1973 and 2012, whereas extrahepatic CCA has seen an increase in incidence from 0.96 to 1.02 per 100,000 over the same time period [90]. The symptoms that patients suffering from CCA present with depends on the type of cancer and its location. Some of the common symptoms include jaundice, pruritis, clay-colored stools, dark urine and other signs of biliary obstruction. The radiological findings present in patients suffering from CCA depend on the imaging modality and the growth pattern of the CCA. If the growth pattern was consistent with mass-forming intrahepatic CCA, an ultrasound would show a homogenous mass with a peripheral hypoechoic halo of compressed liver and the CT would show heterogenous material peripherally with central enhancement $[91,92]$. If the growth pattern was consistent with intraductal tumors, variability in biliary duct diameter and duct ectasia would be seen on CT and ultrasound 91. Figure 3 describes the characteristics of CCA including the subtype of CCA, size, location, pathology, metastatic potential, and symptoms.

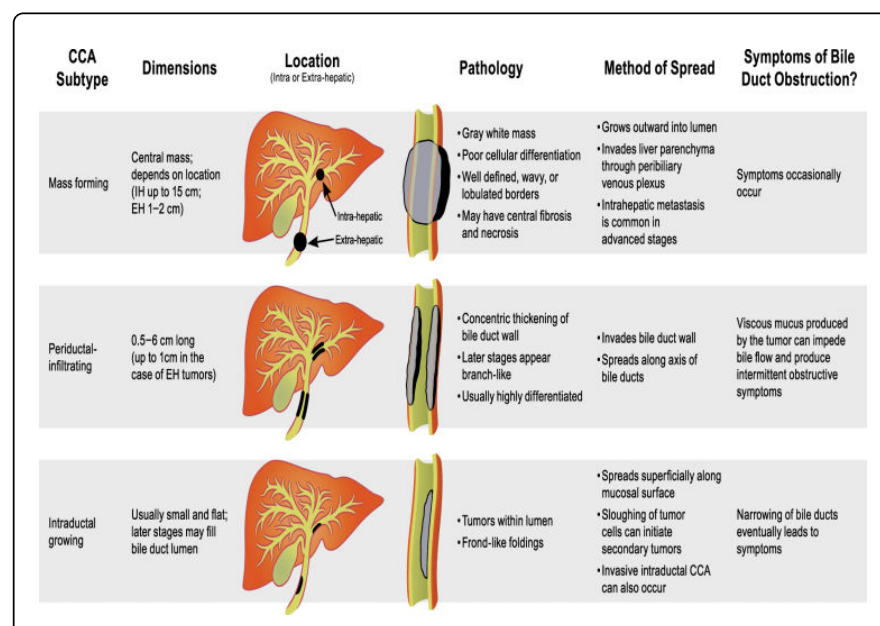

Figure 3: Characteristics of CCA including subtypes, mass formation, periductal infiltration/intraductal, location, pathology, symptoms and methods of metastasis. Adapted from Sripa et al. (2007). Liver fluke induces Cholangiocarcinoma. PLOS Medicine.

Overall, primary liver cancer led to the second most cancer deaths in 2012 [93]. CCA is the second most common form of primary liver cancer, with hepatocellular carcinoma as the leading primary liver cancer [94]. It was reported that the highest rates of development of CCA were in Hispanic and Asian populations (1.8-3.3 per 100,000) with an increased prevalence in males (1.2-1.5 per 100,000) compared to females $(1.0$ per 100,00) [87]. Risk factors for developing CCA include PSC, fibropolycystic liver disease, parasitic infection, viral hepatitis, chronic liver disease, non-alcoholic fatty liver disease, obesity, type 2 diabetes mellitus and other genetic disorders [95,96]. A recent meta-analysis found that there was a moderate correlation between smoking and the development of CCA in Western countries (OR-1.35; CI-1.17 to 1.55) [88]. Another study stated that the increase in risk for developing intrahepatic and extrahepatic CCA was $46 \%$ and $77 \%$, respectively [96]. Creating an environment in which cancer can grow is complex and involves many different changes. A recent article published in Human Pathology studied 54 intrahepatic tumors and found that $7.4 \%$ of cases were found to have mutated KRAS genes and a mutually exclusive $7.4 \%$ were found to have mutated BRAF genes [97]. Both of these genes are involved in the signalling of the RAS/ MAPK pathway that is important for cell signalling and differentiation [97].

MicroRNAs are approximately 22 nucleotide long segments that regulate post-transcriptional regulation of gene expression. Specific 
microRNA segments have been identified to play different roles in the regulations of much pathology [98]. In a published study, the analysis of 80 different liver samples, half of which included tissues with intrahepatic CCA, identified that downregulated microRNA-26b-5p was associated with intrahepatic CCA diagnosis [99]. Additionally, downregulated microRNA-26b-5p correlated with increased invasive capacity of CCA. Administration of microRNA-26b-5p mimics reduced the invasion ability of CCA, and microRNA-26b-5p inhibitors were found to cause increased cell invasion ability. S100 calciumbinding protein A7, which is implicated in the progression of CCA, was found to be a downregulated by microRNA-26b-5p. This is the proposed target that causes the effect of decreased invasion of CCA [99]. MicroRNA-34a is another miRNA that has been found to play a role in the circadian rhythms of humans [100]. This miRNA targets Per1, which inhibits cell growth through various pathways and Per1 is decreased in CCA cells [100].

Inflammation and inflammatory signalling molecules pay an integral role in the development of CCA [101]. Interleukin 6 (IL-6) up regulates myeloid cell leukemia-1 (Mcl-1), a protein that is important in the anti-apoptotic factors in the development of cancer in the biliary epithelial cells [102]. IL-6 has been known to induce epithelial-tomesenchymal transition in CCA [103].

\section{New therapeutic targets}

A 2018 study found that a MAPK inhibitor could be used in the treatment of lung cancers. The use of reactive oxygen species, such as hydrogen peroxide, was found to induce apoptosis in cancer cells. This could be translated to give new guidance in the treatment of CCA [104].

The effects of IL- 6 have been implicated in the prevention of cell apoptosis that allows the development of CCA [97]. Although there are currently medications that are used for this purpose, there are many other drugs that are currently being assessed for safety and effectiveness. These medications are believed to reduce overall inflammation and may assist in alleviating some of the stress that is a precursor to the development of CCA. One such medication, Sarilumab, is an IL-6 receptor blocker that can be used to decrease the downstream effects of IL-6. This medication had demonstrated efficacy in one phase II and six phase III trials as of May 2017 [105].

One of the downstream effects of IL-6 is the upregulation of signal transducers and activators of transcription 3 (STAT3). It was found that activation of epidermal growth factor receptor (EGFR), fibroblast growth factor (FGFR), and platelet-derived growth factor receptor (PDGFR) via phosphorylation leads to activation of STAT3, and a subsequent increase of the cell cycle [106]. In 2009, Sorafenib, a multiple kinase inhibitor, was proven to downregulate the STAT3 pathway [107]. This drug has been known to inhibit EGFR and PDGFR. A 2017 study further analyzed this drug to find that the method of cell death has been found to be linked to cell cycle arrest between the G2 and M phases, leading to apoptosis and inhibition of growth. This was done via the downregulation of a regulatory protein and protein kinase, cyclin B1 and Cdk1, respectively [108].

Nitric oxide synthase is an enzyme found in endothelial cells that plays a role in vasodilation [109]. Interestingly, upregulation of nitric oxide synthase and its modulators has been shown to be correlated with increased angiogenesis and metastasis in CCA [110]. A study showed that inhibition of the nitric oxide synthase using nitro-Larginine methyl ester hydrochloride (L-NAME) resulted in decreased migration and invasion of CCA, with a side effect of hypertension in treated patients [111]. This presents as another potential method for the prevention of CCA progression.

Histamine has been shown to exert pro-tumorigenic effects on CCA progression and angiogenesis [112] and several studies have demonstrated that manipulation of histamine receptors or the enzyme, histidine decarboxylase (HDC), regulates CCA tumor growth. Meng et al. showed that stimulation of H4HR decreased tumor growth both in vivo and in vitro [113] and another study demonstrated that blocking HDC using a-methyl-dl-histidine decreased CCA progression and angiogenesis [60]. Further, and more clinically relevant, a recent study by Kennedy et al. (Figure 2) demonstrated that in addition to blocking PSC [60], chronic antihistamine treatment, via $\mathrm{H} 1 \mathrm{HR}$ or $\mathrm{H} 2 \mathrm{HR}$ inhibition, prevented tumor progression and angiogenesis and reversed EMT in nu/nu mice. Importantly, histamine levels and HDC expression are increased in patients with CCA compared to controls, which might potentially be translated into biomarkers for early diagnosis.

\section{Conclusion}

Given the fine interplay between molecular signalling molecules in the body and biliary tract cholestasis along with the fact that biological technical advances have reached rapid velocities, there will soon be much more information regarding cholangiopathies. By focusing on the molecular mechanisms highlighted in this article, there are many more potential therapeutic targets that need to be further evaluated. If these targets are appropriately vetted, it is possible that effective cures for these cholangiopathies may be discovered.

\section{Conflict of Interest}

The authors have nothing to disclose. This material is the result of work supported with resources and the use of facilities at the Central Texas Veterans Health Care System, Temple, Texas. The content is the responsibility of the author(s) alone and does not necessarily reflect the views or policies of the Department of Veterans Affairs or the United States Government.

\section{Financial support}

Portions of this work were supported by (i) a VA Merit Award (1I01BX003031) from the United States Department of Veteran's affairs, Biomedical Laboratory Research and Development Service and the R01 grant from NIH NIDDK (DK108959).

\section{References}

1. Mousa HS, Lleo A, Invernizzi P, Bowlus CL, Gershwin ME (2015) Advances in pharmacotherapy for primary biliary cirrhosis. Expert Opin Pharmacother 16: 633-643.

2. Zhang LN, Shi TY, Shi XH, Wang L, Yang YJ, et al. (2013) Early biochemical response to ursodeoxycholic acid and long-term prognosis of primary biliary cirrhosis: results of a 14-year cohort study. Hepatology 58: 264-272.

3. Karlsen TH, Vesterhus M, Boberg KM (2014) Review article: controversies in the management of primary biliary cirrhosis and primary sclerosing cholangitis. Aliment Pharmacol Ther 39: 282-301.

4. Lindor KD, Gershwin ME, Poupon R, Kaplan M, Bergasa NV, et al. (2009) Primary biliary cirrhosis. Hepatology 50: 291-308. 
5. Haliloglu N, Erden A, Erden I (2009) Primary biliary cirrhosis: evaluation with T2-weighted MR imaging and MR cholangiopancreatography. Eur Radiol 69: 523-527.

6. Shi TY, Zhang FC (2012) Role of autoimmunity in primary biliary cirrhosis. World J Gastroenterol 18: 7141-7148.

7. Khanna A, Jones DE (2017) Novel strategies and therapeutic options for the management of primary biliary cholangitis. Therap Adv Gastroenterol 10: 791-803.

8. Purohit T, Cappell MS (2015) Primary biliary cirrhosis: Pathophysiology, clinical presentation and therapy. World J Hepatol 7: 926-941.

9. Wang J, Yang GX, Tsuneyama K, Gershwin ME, Ridgway WM (2014) Animal models of primary biliary cirrhosis. Semin Liver Dis 34: 285-296.

10. Corpechot C, Chretien Y, Chazouilleres O, Poupon R (2010) Demographic, lifestyle, medical and familial factors associated with primary biliary cirrhosis. J Hepatol 53: 162-169.

11. Gupta RK, Patel AK, Shah N, Chaudhary AK, Jha UK, et al. (2014) Oxidative stress and antioxidants in disease and cancer: a review. Asian Pac J Cancer Prev 15: 4405-4409.

12. Lushchak (2014) Free radicals, reactive oxygen species, oxidative stress and its classification. Chem Biol Interact 224:164-175.

13. Wink DA, Hines HB, Cheng RY, Switzer CH, Flores-Santana W, et al. (2011) Nitric oxide and redox mechanisms in the immune response. J Leukoc Biol 89: 873-891.

14. Grattagliano I, Calamita G, Cocco T, Wang DQ, Portincasa P (2014) Pathogenic role of oxidative and nitrosative stress in primary biliary cirrhosis. World J Gastroenterol 20: 5746-5759.

15. Rudic JS, Poropat G, Krstic MN, Bjelakovic G, Gluud C (2012) Ursodeoxycholic acid for primary biliary cirrhosis. Cochrane Database Syst Rev12: CD000551.

16. Bolier AR, Peri S, Oude Elferink RP, Beuers U (2012) The challenge of cholestatic pruritus. Acta Gastroenterol Belg 75: 399-404.

17. Mittal A (2016) Cholestatic Itch Management. Curr Probl Dermatol 50: 142-148.

18. Wang L, Li J, Liu H, Li Y, Fu J, et al. (2013) Pilot study of umbilical cordderived mesenchymal stem cell transfusion in patients with primary biliary cirrhosis. J Gastroenterol Hepatol 1: 85-92.

19. Asghar K, Brain J, Palmer JM, Douglass S, Naemi FMA, et al. (2017) Potential role of indoleamine 2,3-dioxygenase in primary biliary cirrhosis. Oncol Lett 14: 5497-5504.

20. Corpechot C, Carrat F, Poujol-Robert A, Gaouar F, Wendum D, et al. (2012) Noninvasive elastography-based assessment of liver fibrosis progression and prognosis in primary biliary cirrhosis. Hepatology 56:198-208.

21. Kuiper EM, Hansen BE, de Vries RA, den Ouden-Muller JW, van Ditzhuijsen TJ, et al. (2009) Improved prognosis of patients with primary biliary cirrhosis that have a biochemical response to ursodeoxycholic acid. Gastroenterology 136: 1281-1287.

22. Lammers WJ, van Buuren HR, Hirschfield GM, Janssen HL, Invernizzi P, et al. (2014) Levels of alkaline phosphatase and bilirubin are surrogate end points of outcomes of patients with primary biliary cirrhosis: an international follow-up study. Gastroenterology 147: 1338-1349 e1335.

23. Angulo P, Lindor KD (1999) Primary sclerosing cholangitis. Hepatology 30: 325-332.

24. Lee YM, Kaplan MM (1995) Primary sclerosing cholangitis. N Engl J Med 332: 924-933.

25. Rupp C, Rossler A, Zhou T, Rauber C, Friedrich K, et al. (2018) Impact of age at diagnosis on disease progression in patients with primary sclerosing cholangitis. United European Gastroenterol J 6: 255-262.

26. Molodecky NA, Kareemi H, Parab R, Barkema HW, Quan H, et al. (2011) Incidence of primary sclerosing cholangitis: a systematic review and meta-analysis. Hepatology 53: 1590-1599.

27. Boonstra K, Weersma RK, van Erpecum KJ, Rauws EA, Spanier BW, et al. (2013) Population-based epidemiology, malignancy risk, and outcome of primary sclerosing cholangitis. Hepatology 58: 2045-2055.
28. Sorensen JO, Nielsen OH, Andersson M, Ainsworth MA, Ytting H, et al. (2018) Inflammatory bowel disease with primary sclerosing cholangitis: A Danish population-based cohort study 1977-2011. Liver Int 38: 532-541.

29. Shah SC, Ten Hove JR, Castaneda D, Palmela C, Mooiweer E, et al. (2018) High Risk of Advanced Colorectal Neoplasia in Patients With Primary Sclerosing Cholangitis Associated With Inflammatory Bowel Disease. Clin Gastroenterol Hepatol 16: 1106-1113 e1103.

30. Kummen M, Schrumpf E, Boberg KM (2013) Liver abnormalities in bowel diseases. Best Pract Res Clin Gastroenterol 27: 531-542.

31. de Vries AB, Janse M, Blokzijl H, Weersma RK (2015) Distinctive inflammatory bowel disease phenotype in primary sclerosing cholangitis. World J Gastroenterol 21: 1956-1971.

32. Nakazawa T, Naitoh I, Hayashi K, Sano H, Miyabe K, et al. (2014) Inflammatory bowel disease of primary sclerosing cholangitis: a distinct entity? World J Gastroenterol 20: 3245-3254.

33. Ali AH, Tabibian JH, Nasser-Ghodsi N, Lennon RJ, DeLeon $\mathrm{T}$ et al. (2018) Surveillance for hepatobiliary cancers in patients with primary sclerosing cholangitis. Hepatology 67: 2338-2351.

34. Broome U, Olsson R, Loof L, Bodemar G, Hultcrantz R, et al. (1996) Natural history and prognostic factors in 305 Swedish patients with primary sclerosing cholangitis. Gut 38: 610-615.

35. Tischendorf JJ, Hecker H, Kruger M, Manns MP, Meier PN (2007) Characterization, outcome, and prognosis in 273 patients with primary sclerosing cholangitis: A single center study. Am J Gastroenterol 102: 107-114.

36. Lindor KD, Kowdley KV, Harrison ME, American College of G (2015) ACG Clinical Guideline: Primary Sclerosing Cholangitis. Am J Gastroenterol 110: 646-659.

37. Hov JR, Boberg KM, Karlsen TH (2008) Autoantibodies in primary sclerosing cholangitis. World J Gastroenterol 14: 3781-3791.

38. Marchioni Beery RM, Vaziri H, Forouhar F (2014) Primary Biliary Cirrhosis and Primary Sclerosing Cholangitis: a Review Featuring a Women's Health Perspective. J Clin Transl Hepatol 2: 266-284.

39. Chapman R, Fevery J, Kalloo A, Nagorney DM, Boberg KM, et al. (2010) Diagnosis and management of primary sclerosing cholangitis. Hepatology 51: 660-678.

40. Lindor KD (1997) Ursodiol for primary sclerosing cholangitis. Mayo Primary Sclerosing Cholangitis-Ursodeoxycholic Acid Study Group. N Engl J Med 336: 691-695.

41. Lindor KD, Kowdley KV, Luketic VA, Harrison ME, McCashland T, et al. (2009) High-dose ursodeoxycholic acid for the treatment of primary sclerosing cholangitis. Hepatology 50: 808-814.

42. Triantos CK, Koukias NM, Nikolopoulou VN, Burroughs AK (2011) Meta-analysis: ursodeoxycholic acid for primary sclerosing cholangitis. Aliment Pharmacol Ther 34: 901-910.

43. Borody TJ, Brandt LJ, Paramsothy S (2014) Therapeutic faecal microbiota transplantation: current status and future developments. Curr Opin Gastroenterol 30: 97-105.

44. Davies YK, Tsay CJ, Caccamo DV, Cox KM, Castillo RO, et al. (2013) Successful treatment of recurrent primary sclerosing cholangitis after orthotopic liver transplantation with oral vancomycin. Case Rep Transplant p:314292.

45. Gautam M, Cheruvattath R, Balan V (2006) Recurrence of autoimmune liver disease after liver transplantation: a systematic review. Liver Transpl 12: $1813-1824$.

46. Campsen J, Zimmerman MA, Trotter JF, Wachs M, Bak T, et al. (2008) Clinically recurrent primary sclerosing cholangitis following liver transplantation: a time course. Liver Transpl 14:181-185.

47. Trivedi PJ, Chapman RW (2012) PSC, AIH and overlap syndrome in inflammatory bowel disease. Clin Res Hepatol Gastroenterol 36: 420-436.

48. Brenchley JM, Douek DC (2012) Microbial translocation across the GI tract. Annu Rev Immunol 30: 149-173.

49. Karlsen TH, Boberg KM (2013) Update on primary sclerosing cholangitis. J Hepatol 59:571-582. 
50. Tabibian JH, O'Hara SP, Splinter PL, Trussoni CE, LaRusso NF (2014) Cholangiocyte senescence by way of N-ras activation is a characteristic of primary sclerosing cholangitis. Hepatology 59: 2263-2275.

51. Tabibian JH, Trussoni CE, O'Hara SP, Splinter PL, Heimbach JK, et al. (2014) Characterization of cultured cholangiocytes isolated from livers of patients with primary sclerosing cholangitis. Lab Invest 94:1126-1133.

52. Eaton JE, Talwalkar JA, Lazaridis KN, Gores GJ, Lindor KD (2013) Pathogenesis of primary sclerosing cholangitis and advances in diagnosis and management. Gastroenterology 145: 521-536.

53. Trivedi PJ, Hirschfield GM (2013) Treatment of autoimmune liver disease: current and future therapeutic options. Ther Adv Chronic Dis 4: 119-141.

54. Fickert P, Hirschfield GM, Denk G, Marschall HU, Altorjay I, et al. (2017) norUrsodeoxycholic acid improves cholestasis in primary sclerosing cholangitis. J Hepatol 67: 549-558.

55. Francis H, Meininger CJ (2010) A review of mast cells and liver disease: What have we learned? Dig Liver Dis 42: 529-536.

56. Koruk ST, Ozardali I, Dincoglu D, Bitiren M (2011) Increased liver mast cells in patients with chronic hepatitis C. Indian J Pathol Microbiol 54: 736-740.

57. Kennedy LL, Hargrove LA, Graf AB, Francis TC, Hodges KM, et al. (2014)Inhibition of mast cell-derived histamine secretion by cromolyn sodium treatment decreases biliary hyperplasia in cholestatic rodents. Lab Invest. 94:1406-1418.

58. Jones H, Hargrove L, Kennedy L, Meng F, Graf-Eaton A, et al. (2016) Inhibition of mast cell-secreted histamine decreases biliary proliferation and fibrosis in primary sclerosing cholangitis Mdr2(-/-) mice. Hepatology 64:1202-1216.

59. Osterreicher $\mathrm{CH}$, Trauner M (2012) Animal models of biliary tract injury. Curr Opin Gastroenterol 28: 239-243.

60. Kennedy L, Hargrove L, Demieville J, Karstens W, Jones H, et al. (2018) Blocking $\mathrm{H} 1 / \mathrm{H} 2$ histamine receptors inhibits damage/fibrosis in $\operatorname{Mdr2(-/-)}$ mice and human cholangiocarcinoma tumorigenesis. Hepatology.

61. Peng X, Luo X, Hou JY, Wu SY, Li LZ, et al. (2017) Immunosuppressive Agents for the Treatment of Primary Sclerosing Cholangitis: A Systematic Review and Meta-Analysis. Dig Dis 35: 478-485.

62. Tajiri H, Zen Y, Takano T, Brooks S (2018) Favorable response to immunosuppressive combination therapy with mizoribine and azathioprine in children with primary sclerosing cholangitis. Hepatol Res 48: 322-328.

63. Westerveld D, Grajo J, Beattie L, Glover S (2017) Vedolizumab: a novel medical intervention in the treatment of primary sclerosing cholangitis. BMJ Case Rep.

64. Czaja AJ (2013) The overlap syndromes of autoimmune hepatitis. Dig Dis Sci 58: 326-343.

65. Rojas CP, Bodicharla R, Campuzano-Zuluaga G, Hernandez L, Rodriguez MM (2013) Autoimmune hepatitis and primary sclerosing cholangitis in children and adolescents. Fetal Pediatr Pathol 33: 202-209.

66. Lee S, Park H, Moon SB, Jung SM, Kim JM, et al. (2013) Long-term results of biliary atresia in the era of liver transplantation. Pediatr Surg Int 29: 1297-1301.

67. Verkade HJ, Bezerra JA, Davenport M, Schreiber RA, Mieli-Vergani G, et al. (2016) Biliary atresia and other cholestatic childhood diseases: Advances and future challenges. J Hepatol 65: 631-642.

68. Govindarajan KK (2016) Biliary atresia: Where do we stand now? World J Hepatol 8: 1593-1601.

69. Nizery L, Chardot C, Sissaoui S, Capito C, Henrion-Caude A, et al. (2016) Biliary atresia: Clinical advances and perspectives. Clin Res Hepatol Gastroenterol 40: 281-287.

70. Hopkins PC, Yazigi N, Nylund CM (2017) Incidence of Biliary Atresia and Timing of Hepatoportoenterostomy in the United States. J Pediatr 87: 253-257.

71. Lakshminarayanan B, Davenport M (2016) Biliary atresia: A comprehensive review. J Autoimmun73: 1-9.
72. Lee HJ, Lee SM, Park WH, Choi SO (2003) Objective criteria of triangular cord sign in biliary atresia on US scans. Radiology 229: 395-400.

73. Sanchez-Valle A, Kassira N, Varela VC, Radu SC, Paidas C, et al. (2017) Biliary Atresia: Epidemiology, Genetics, Clinical Update, and Public Health Perspective. Adv Pediatr 64: 285-305.

74. Shivakumar P, Mourya R, Bezerra JA (2014) Perforin and granzymes work in synergy to mediate cholangiocyte injury in experimental biliary atresia. J Hepatol 60: 370-376.

75. Okamura A, Harada K, Nio M, Nakanuma Y (2013) Interleukin-32 production associated with biliary innate immunity and proinflammatory cytokines contributes to the pathogenesis of cholangitis in biliary atresia. Clin Exp Immunol 173: 268-275.

76. Shivakumar P, Mizuochi T, Mourya R, Gutta S, Yang L, et al. (2017) Preferential TNFalpha signaling via TNFR2 regulates epithelial injury and duct obstruction in experimental biliary atresia. JCI Insight 2: e88747.

77. Hong SM, Park IH, Um JY, Shin JM, Lee HM (2015) Stimulatory effects of histamine on migration of nasal fibroblasts. Int Forum Allergy Rhinol 5: 923-928.

78. Levick SP, Widiapradja A (2018) Mast Cells: Key Contributors to Cardiac Fibrosis. Int J Mol Sci19.

79. Zhou K, Xie G, Wen J, Wang J, Pan W, et al. (2016) Histamine is correlated with liver fibrosis in biliary atresia. Dig Liver Dis 48: 921-926.

80. Kishore R, Kisku SMC, Thomas RJ, Jeenipalli SK (2018) Laparoscopic cholangiogram in biliary atresia: a refinement in the gallbladder hitch technique. Pediatr Surg Int 34: 395-398.

81. Lishuang M, Zhen C, Guoliang Q, Zhen Z, Chen W, et al. (2015) Laparoscopic portoenterostomy versus open portoenterostomy for the treatment of biliary atresia: a systematic review and meta-analysis of comparative studies. Pediatr Surg Int 31: 261-269.

82. Cazares J, Koga H, Murakami H, Nakamura H, Lane G, et al. (2017) Laparoscopic portoenterostomy for biliary atresia: single-center experience and review of literatures. Pediatr Surg Int 33: 1341-1354.

83. Lorent K, Gong W, Koo KA, Waisbourd-Zinman O, Karjoo S, et al. (2015) Identification of a plant isoflavonoid that causes biliary atresia. Sci Transl Med 7: $286 \mathrm{ra} 267$.

84. Bassalert C, Valverde-Estrella L, Chazaud C (2018) Primitive Endoderm Differentiation: From Specification to Epithelialization. Curr Top Dev Biol 128: 81-104.

85. Zhao X, Zhang W, Ji W (2018)miR-181a targets GATA6 to inhibit the progression of human laryngeal squamous cell carcinoma. Future Oncol.

86. Soini T, Pihlajoki M, Andersson N, Lohi J, Huppert KA, et al. (2018) Transcription factor GATA6: a novel marker and putative inducer of ductal metaplasia in biliary atresia. Am J Physiol Gastrointest Liver Physiol 314: G547-G558.

87. Razumilava N, Gores GJ (2014) Cholangiocarcinoma. Lancet 383: 2168-2179.

88. Huang Y, You L, Xie W, Ning L, Lang J (2017) Smoking and risk of cholangiocarcinoma: a systematic review and meta-analysis. Oncotarget 8:100570-100581.

89. DeOliveira ML, Cunningham SC, Cameron JL, et al. (2007) Cholangiocarcinoma: thirty-one-year experience with 564 patients at a single institution. Ann Surg 245: 755-762.

90. Oliveira IS, Kilcoyne A, Everett JM, Mino-Kenudson M, Harisinghani MG, et al. (2017) Cholangiocarcinoma: classification, diagnosis, staging, imaging features, and management. Abdom Radiol (NY) 42: 1637-1649.

91. Chung YE, Kim MJ, Park YN, Choi JY, Pyo JY, et al. (2009) Varying appearances of cholangiocarcinoma: radiologic-pathologic correlation. Radiographics 29: 683-700.

92. Han JK, Choi BI, Kim AY, Kyung An S, Lee JW, et al. (2002) Cholangiocarcinoma: pictorial essay of $\mathrm{CT}$ and cholangiographic findings. Radiographics 22: 173-187.

93. Ferlay J, Soerjomataram I, Dikshit R, Eser S, Mathers C, et al. (2015) Cancer incidence and mortality worldwide: sources, methods and major patterns in Globocan 2012. Int J Cancer136: E359-386. 
Citation: Virani S, Akers A, Stephenson K, Smith S, Kennedy L, et al. (2018) Comprehensive Review of Molecular Mechanisms during Cholestatic Liver Injury and Cholangiocarcinoma. J Liver 7: 231. doi:10.4172/2167-0889.1000231

Page 10 of 10

94. Massarweh NN, El-Serag HB (2017) Epidemiology of Hepatocellular Carcinoma and Intrahepatic Cholangiocarcinoma. Cancer Control 24: 1073274817729245

95. Garg R, Khan U, AlRajjal A, Kafri Z (2017) Autoimmune Hepatitis: A Risk Factor for Cholangiocarcinoma. Case Rep Gastroenterol11: 672-677.

96. Petrick JL, Yang B, Altekruse SF, Van Dyke AL, Koshiol J, et al. (2017) Risk factors for intrahepatic and extrahepatic cholangiocarcinoma in the United States: A population-based study in SEER-Medicare. PLoS One 12: e0186643.

97. Robertson S, Hyder O, Dodson R, Nayar SK, Poling J, et al. (2013) The frequency of KRAS and BRAF mutations in intrahepatic cholangiocarcinomas and their correlation with clinical outcome. Hum Pathol 44: 2768-2773.

98. Bartel DP (2004) MicroRNAs: genomics, biogenesis, mechanism, and function. Cell 116: 281-297.

99. Fan F, Lu J, Yu W, Zhang Y, Xu S, et al. (2018) MicroRNA-26b-5p regulates cell proliferation, invasion and metastasis in human intrahepatic cholangiocarcinoma by targeting S100A7. Oncol Lett 15: 386-392.

100. Han Y, Meng F, Venter J, Wu N, Wan Y, et al. (2016) miR-34a-dependent overexpression of Per1 decreases cholangiocarcinoma growth. J Hepatol 64: 1295-1304.

101. Maemura K, Natsugoe S, Takao S (2014) Molecular mechanism of cholangiocarcinoma carcinogenesis. J Hepatobiliary Pancreat Sci 21: 754-760.

102. Isomoto H, Kobayashi S, Werneburg NW, Bronk SF, Guicciardi ME, et al. (2005) Interleukin 6 upregulates myeloid cell leukemia-1 expression through a STAT3 pathway in cholangiocarcinoma cells. Hepatology 42:1329-1338.

103. Zhou QX, Jiang XM, Wang ZD, Li CL, Cui YF (2015)Enhanced expression of suppresser of cytokine signaling 3 inhibits the IL-6-induced epithelial-to-mesenchymal transition and cholangiocarcinoma cell metastasis. Med Oncol 32:105.

104. Park WH (2018) MAPK inhibitors, particularly the JNK inhibitor increase cell death effects in $\mathrm{H} 2 \mathrm{O} 2$-treated lung cancer cells via increased superoxide anion and glutathione depletion. Oncol Rep 39: 860-870.
105. Kretsos K, Golor G, Jullion A, Hickling M, McCabe S, et al. (2014) Safety and pharmacokinetics of olokizumab, an anti-IL-6 monoclonal antibody, administered to healthy male volunteers: A randomized phase I study. Clin Pharmacol Drug Dev 3: 388-395.

106. Schuringa JJ, Wierenga AT, Kruijer W, Vellenga E (2000) Constitutive Stat3, Tyr705, and Ser727 phosphorylation in acute myeloid leukemia cells caused by the autocrine secretion of interleukin-6. Blood 95: 3765-3770.

107. Blechacz BR, Smoot RL, Bronk SF, Werneburg NW, Sirica AE, et al. (2009) Sorafenib inhibits signal transducer and activator of transcription-3 signaling in cholangiocarcinoma cells by activating the phosphatase shatterproof 2. Hepatology 50: 1861-1870.

108. Hu MH, Chen LJ, Chen YL, Tsai MS, Shiau CW, et al. (2017) Targeting SHP-1-STAT3 signaling: A promising therapeutic approach for the treatment of cholangiocarcinoma. Oncotarget 8: 65077-65089.

109. Yang Y, Yu T, Lian YJ, Ma R, Yang S, et al. (2015) Nitric oxide synthase inhibitors: a review of patents from 2011 to the present. Expert Opin Ther Pat 25: 49-68.

110. Suksawat M, Techasen A, Namwat N, Yongvanit P, Khuntikeo N, et al. (2017) Upregulation of endothelial nitric oxide synthase (eNOS) and its upstream regulators in Opisthorchis viverrini associated cholangiocarcinoma and its clinical significance. Parasitol Int 66: 486-493.

111. Suksawat M, Techasen A, Namwat N, Boonsong T, Titapun A, et al. (2018) Inhibition of endothelial nitric oxide synthase in cholangiocarcinoma cell lines - a new strategy for therapy. FEBS Open Bio 8: 513-522.

112. Francis H, DeMorrow S, Venter J, Onori P, White M, et al. (2012) Inhibition of histidine decarboxylase ablates the autocrine tumorigenic effects of histamine in human cholangiocarcinoma. Gut 61: 753-764.

113. Meng F, Han Y, Staloch D, Francis T, Stokes A, et al. (2011) The H4 histamine receptor agonist, clobenpropit, suppresses human cholangiocarcinoma progression by disruption of epithelial mesenchymal transition and tumor metastasis. Hepatology 54: 1718-1728. 